\title{
CircSLC3A2 functions as an oncogenic factor in hepatocellular carcinoma by sponging miR-490-3p and regulating PPM1F expression
}

\author{
Hongjian Wang ${ }^{1}$, Wei Chen², Ming Jin ${ }^{3}$, Lidan Hou ${ }^{4}$, Xiaoyu Chen², Rui Zhang ${ }^{2}$, Jing Zhang ${ }^{2 *}$ and Jinshui Zhu
}

\begin{abstract}
Background: Non-coding RNAs (ncRNAs) have been reported to participate in tumor progression by regulating gene expression. Previous studies showed that protein phosphatase $\mathrm{Mg}^{+} / \mathrm{Mn2}^{+}$dependent $1 \mathrm{~F}$ (PPM1F) acts a dual role in cancer growth and metastasis. But, the underlying mechanisms by which ncRNAs regulate PPM1F expression in hepatocellular carcinoma (HCC) are poorly understood.

Methods: The association between PPM1F or miR-490-3p expression and clinicopathological features and prognosis in patients with HCC was analyzed by TCGA RNA-sequencing data. CircSLC3A2 was identified to bind with miR-490-3p by bioinformatic analysis, and the binding sites between miR-490-3p and PPM1F or circSLC3A2 were confirmed by dual luciferase report and RNA immunoprecipitation (RIP) assays. The localization and clinical significance of miR-490-3p and circSLC3A2 in patients with HCC were investigated by fluorescence in situ hybridization (FISH). MTT, Agar, and Transwell assays were conducted to evaluate the effects of miR-490-3p or circSLC3A2 on cell proliferation and invasive potential.

Results: The expression of PPM1F or miR-490-3p was associated with poor survival and tumor recurrence, and acted as an independent prognostic factor in patients with HCC. Re-expression of miR-490-3p inhibited HCC cell proliferation and invasion by targeting PPM1F, but its inhibitor reversed these effects. Moreover, circSLC3A2, predominantly localized in the cytoplasm, exhibited an oncogenic role by sponging miR-490-3p and regulating PPM1F expression, and harbored a positive correlation with poor survival in patients with HCC.
\end{abstract}

Conclusion: CirCSLC3A2 acts as an oncogenic factor in HCC by sponging miR-490-3p and regulating PPM1F expression.

Keywords: PPM1F, CircSLC3A2, MiR-490-3p, Proliferation, Invasion, Hepatocellular carcinoma

\section{Introduction}

Hepatocellular carcinoma (HCC) is the second leading cause of cancer-related death and its incidence is increasing each year worldwide [1]. Despite the feasible and effective therapies for HCC, such as surgical resection, ablation, chemotherapy and liver transplantation, the prognosis of patients with HCC remains very poor duo to its aggressiveness and diagnosis at a late stage [2]. HCC is a long-term progressive disease with multiple genetic

\footnotetext{
* Correspondence: jing5522724@vip.163.com

${ }^{2}$ Department of Gastroenterology, Shanghai Jiao Tong University Affiliated Sixth People's Hospital, No. 600 Yishan Road, Shanghai 200233, China Full list of author information is available at the end of the article
}

alterations, leading to the activation of oncogenes or inactivation of tumor suppressor genes [3]. Therefore, identification of novel promising biomarkers may provide insights into the early diagnosis of $\mathrm{HCC}$.

Protein phosphatase, $\mathrm{Mg}^{+} / \mathrm{Mn}^{+}$dependent $1 \mathrm{~F}$ (PPM1F), a member of the PP2C family of Ser/Thr protein phosphatases, has been reported to regulate cancer cell apoptosis, proliferation and metastasis. PPM1F, expressed in a variety of tumor cell lines, facilitates cell motility and invasiveness [4] and breast carcinogenesis by inactivating p53 signaling [5], promotes tumor metastasis via MAPK signaling and exosome cytokine secretion [6], and represses cell apoptosis through the TAK1-IKK-NF- $\mathrm{KB}$

(c) The Author(s). 2018 Open Access This article is distributed under the terms of the Creative Commons Attribution 4.0 International License (http://creativecommons.org/licenses/by/4.0/), which permits unrestricted use, distribution, and 
pathway [7]. However, our previous study showed that PPM1F is downregulated in gastric cancer (GC), and low expression of PPM1F is associated with poor survival in patients with GC [8].

MicroRNAs (miRNAs) are a subgroup of endogenous non-coding RNAs (ncRNAs) with 20 25 nucleotides in length and function by negatively regulating their target genes [9]. Accumulating evidence indicates that miRNAs can act as oncogenic or suppressive factors involved in the progression of HCC [10-12], and some even exhibit antitumor effects by regulating PPM1F expression. For example, miR-200c and miR-149 inhibit the invasion and metastasis in breast cancer and HCC by targeting PPM1F [13, 14], but miR-590 has the tumor promoting effects in GC by targeting PPM1F [8].

Circular RNAs (circRNAs) as another new class of ncRNAs possess a closed loop and higher conservatism than linear RNA duo to their resistance to RNase R [15]. Increasing data show that circRNAs, predominantly localized in the cytoplasm, can act as the miRNA sponges to participate in the pathogenesis of HCC [16]. CircRNA cSMARCA5 and circMTO1, associated with tumor aggressive characteristics, suppress the growth and metastasis of HCC by sponging miR-17-3p, miR-181b-5p and miR-9 [17, 18], whereas circ_0067934 favors the growth and metastasis of HCC by regulating miR-1324/FZD5/ Wnt/ $\beta$-catenin signaling [19]. These studies suggest that circRNAs may represent novel biomarkers for the diagnosis and treatment of HCC.

In this study, we found that the expression of PPM1F or miR-490-3p was associated with poor survival and tumor recurrence, and acted as an independent prognostic factor in patients with HCC. Then, miR-490-3p was identified to have a negative correlation with PPM1F expression and inhibited cell proliferation and invasion by targeting PPM1F. CircSLC3A2 was further found to function as an oncogenic factor in HCC cells by sponge miR-490-3p and regulating PPM1F expression, and was associated with the poor survival in patients with HCC.

\section{Materials \& methods}

\section{Clinical data}

The clinicopathological data of 50 paired HCC and adjacent non-tumor tissues as well as 372 unpaired HCC tissues, the relative expression levels of PPM1F and miRNAs (has-miR-186-5p, has-miR-200c, has-miR-200b, has-miR-429, has-miR-425-5p and has-miR-490-3p) were downloaded from The Cancer Genome Atlas (TCGA)-Liver Cancer RNA sequencing database (https://genome-cancer.ucsc.edu). The tissue microarray (TMA) of 90 paired HCC patients (Cat No. K16-027) was purchased from the shanghai Outdo Biotech Company (Shanghai, PR, China). The protocols used in our study were approved by the Ethics Committee of Shanghai Sixth People's Hospital. HCC specimens were classified according to the WHO criteria and TNM staging system, and the pathological diagnosis for these samples was accomplished by two independent pathologists.

\section{Identification of miRNAs that target PPM1F 3'UTR}

The miRNAs that target PPM1F 3'UTR were identified using both the TargetScan_7.1 (http://www.targetscan.org/vert_71/) and microRNA.org (http://starbase.sysu.edu.cn/targetSite.php), and the intersecting miRNAs from these two prediction tools were used for further verification.

\section{Cell culture}

Normal liver tissue and HCC cell lines (HepG2, Huh6, Huh7, SK-hep-1, SMMC-7721 and LO2) were from Cell bank of the Chinese Academy of Sciences and cultured in Dulbecco's Modified Eagle medium (DMEM) medium supplemented with $10 \%$ heat-inactivated fetal bovine serum (FBS). Cells in this medium were placed in a humidified atmosphere containing $5 \% \mathrm{CO}_{2}$ at $37{ }^{\circ} \mathrm{C}$.

\section{RNA fluorescence in situ hybridization (FISH)}

Oligonucleotide modified probe sequences for miR-490-3p (5' -CY3-CAGCATGGAG TCCTCCAGG TTG-CY3-3) and circSLC3A2 (5'-FAM-GTAGTTGGG AGTAAG GT CCAGAATGACACGGATGCCTGTCC AGGAA-FAM- $3^{\prime}$.) were used for FISH. The detailed experimental process was performed as previously described [20]. The analysis software Image-pro plus 6.0 (Media Cybernetics, Inc., Rockville, MD, USA) was used to analyse the Immunofluorescence Accumulation Optical Density (IOD) of miR-490-3p and circSLC3A2 in HCC tissues.

\section{Quantitative real-time PCR (qRT-PCR)}

Total RNA was extracted using TRIzol and reverse transcription was conducted using M-MLV and cDNA amplification using the SYBR Green Master Mix kit (Takara, Otsu, Japan). In addition, total RNA was separated using a High Pure miRNA isolation kit (Roche) and RT-PCR using a TaqMan MicroRNA Reverse Transcription kit (Life Technologies). The nuclear and cytoplasmic fractions were isolated using NE-PER Nuclear and Cytoplasmic Extraction Reagents (Thermo Scientific). The primer sequences were indicated in Additional file 1: Table S1.

\section{Western blotting analysis}

HepG2 and LO2 cell lines were harvested and extracted using lysis buffer. Cell extracts were boiled in loading buffer and equal amount of cell extracts were separated on $15 \%$ SDS-PAGE gels. Separated protein bands were transferred into polyvinylidene fluoride (PVDF) membranes. 
The primary antibodies against PPM1F (ab156222, Rabbit polyclonal antibody, Abcam, Cambridge, MA, USA) and $\beta$-actin (ab16039, Rabbit polyclonal antibody, Abcam, Cambridge, MA, USA) were diluted at a ratio of 1:1000 according to the instructions and incubated overnight at $4{ }^{\circ} \mathrm{C}$. Horseradish peroxidase-linked secondary antibodies were added at a dilution ratio of 1:10000, and incubated at room temperature for $1 \mathrm{~h}$. The membranes were washed with PBS for three times and the immunoreactive bands were visualized using ECL-PLUS/Kit (GE Healthcare, Piscataway, NJ, USA) according to the kit's instruction.

\section{Luciferase reporter assay}

HepG2 and LO2 cell lines were seeded into 96-well plates and were co-transfected with a mixture of $60 \mathrm{ng}$ of luciferase, $6 \mathrm{ng}$ of pRL-CMV Renilla luciferase reporter, and miR-490-3p mimic or inhibitor. After $48 \mathrm{~h}$ of incubation, the firefly and Renilla luciferase activities were measured with a dual-luciferase reporter assay (Promega, Madison, WI, USA).

\section{Plasmid, shRNA, miRNA mimic and inhibitor}

Plasmid mediated PPM1F or circSLC3A2 vector, RNAi targeting PPM1F or circSLC3A2 vector, miR-490-3p mimic and inhibitor were purchased from GenePharma (Shanghai, PR, China) and the negative control (NC), pcDNA3.1 or miR-NC was used as the control vector. Designed RNAi sequences were listed as follows: si-PPM1F (NM 014634 siRNA 405): GCTGCTACA GACAGACCTT and NM_014634_siRNA_control_405: GCTACATACAGCAGCGCTT; sh-circSLC3A2: GAC AGGCATCCGTGTCATTCT and sh-NC: GCTC ACTTAGTTATCGGAC. HepG2 and LO2 cell lines were planted in 6-well plates $24 \mathrm{~h}$ prior to si-PPM1F, sh-circSLC3A2, miR-490-3p mimic or inhibitor transfection with $50-60 \%$ confluence, and then were transfected

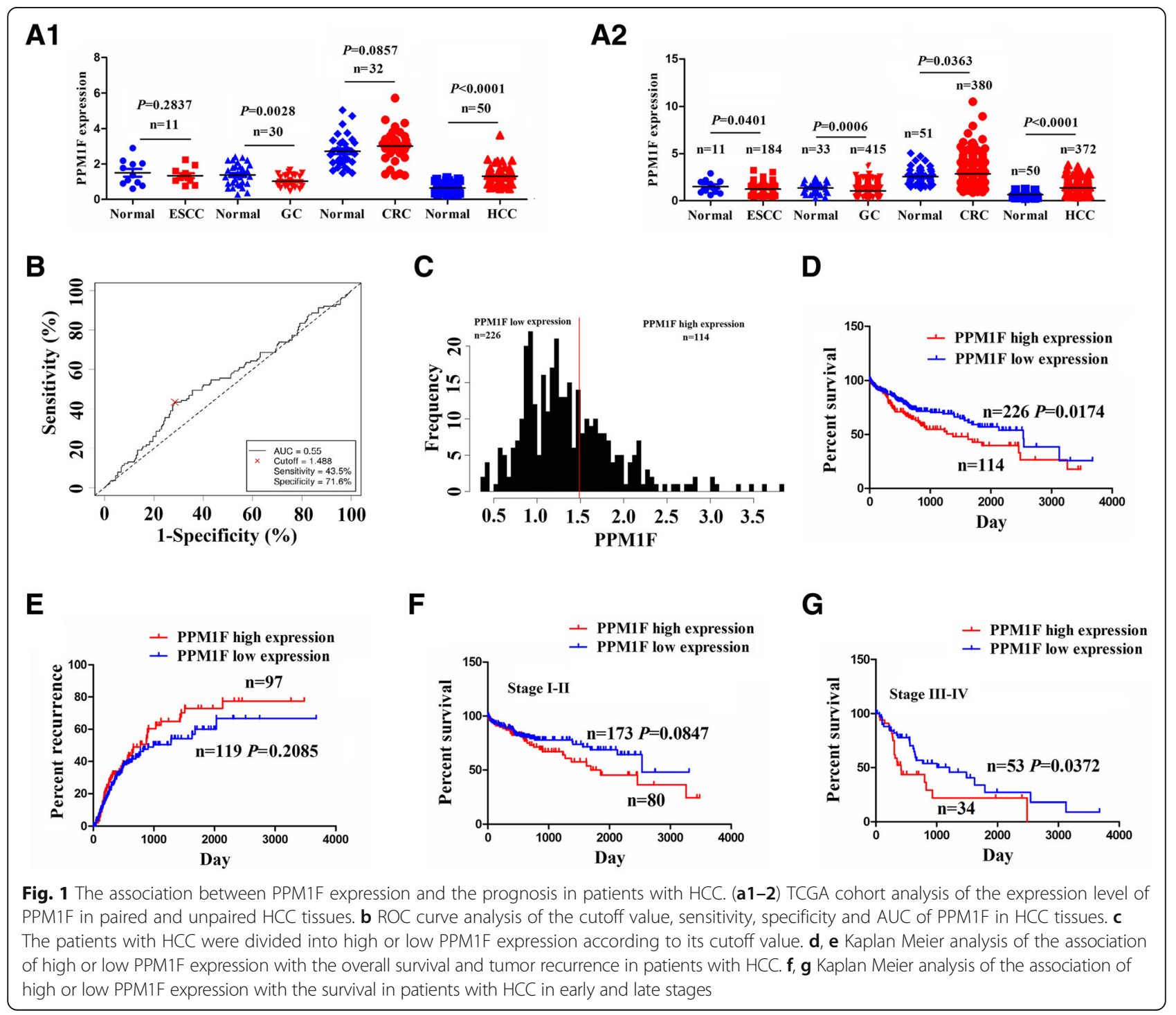


with Lipofectamine 2000 (Invitrogen, Carlsbad, CA, USA) according to the manufacture instructions.

\section{MTT and Transwell assays}

MTT assay for cell viability and Transwell assay for cell invasion were conducted as previously described [20].

\section{Agar assay}

Cells were trypsinized, and 5000 cells were resuspended in $2 \times$ medium plus $0.7 \%$ agar (Sigma). The agar-cell mixture was plated on top of a bottom layer consisting of $1.2 \%$ agar in complete medium. After 10 days, colony size was measured using an ocular micrometer and colonies larger than $0.1 \mathrm{~mm}$ in diameter were counted. The experiment was performed three times for each cell line.

\section{RNase R treatment}

Total RNA $(2 \mu \mathrm{g})$ was incubated for $30 \mathrm{~min}$ at $37^{\circ} \mathrm{Cwith}$ $3 \mathrm{U} / \mu \mathrm{g}$ of RNase R (Epicentre Technologies, Madison, WI, USA). After treatment with RNase $\mathrm{R}$, the mRNA levels of SLC3A2 and circSLC3A2 were examined by qRT-PCR analysis.

\section{RNA immunoprecipitation (RIP)}

RIP assay was performed using a Magna RIP RNA-binding protein Immunoprecipitation Kit (Millipore) according to the manufacturer's instructions. Antibodies for RIP assays against Ago2 and IgG were purchased from Abcam (ab5072, Rabbit polyclonal antibody, Cambridge, MA, USA).

\section{Statistical analysis}

Statistical analyses were implemented using SPSS 20.0 (IBM, SPSS, Chicago, IL, USA) and GraphPad Prism. Student's t-test and Chi-square test were used to analyze the statistical significance for comparisons of two groups. The Pearson's correlation coefficient analysis was used to observe the correlations between miRNAs and PPM1F or circSLC3A2 expression in HCC tissues. Overall survival and disease-free survival (DFS or recurrence) curves were analyzed with Kaplan-Meier method and log-rank test. Univariate analysis and multivariate models were conducted using a Cox proportional hazards regression model. Cutoff value and Receiver operating characteristic (ROC) curve were obtained using cutoff finder online software (http://molpath.charite.de/cutoff/load.jsp). $P<$ 0.05 was considered statistically significant.

\section{Results}

Upregulation of PPM1F expression was associated with poor survival in patients with HCC

We examined the expression levels of PPM1F in multiple gastrointestinal tumors using the TCGA database and found that PPM1F had the most obvious upregulation in paired $(n=50, P<0.0001$; Fig. 1a1) and unpaired HCC tissues $(n=372, P<0.0001$; Fig. 1a2) as compared with the other tumor tissues. To assess the clinical significance of PPM1F in HCC, we analyzed the association between PPM1F expression and clinicopathological characteristics and prognosis in patients with HCC. As shown in Fig. 1b, the cutoff value of PPM1F (1.488) was

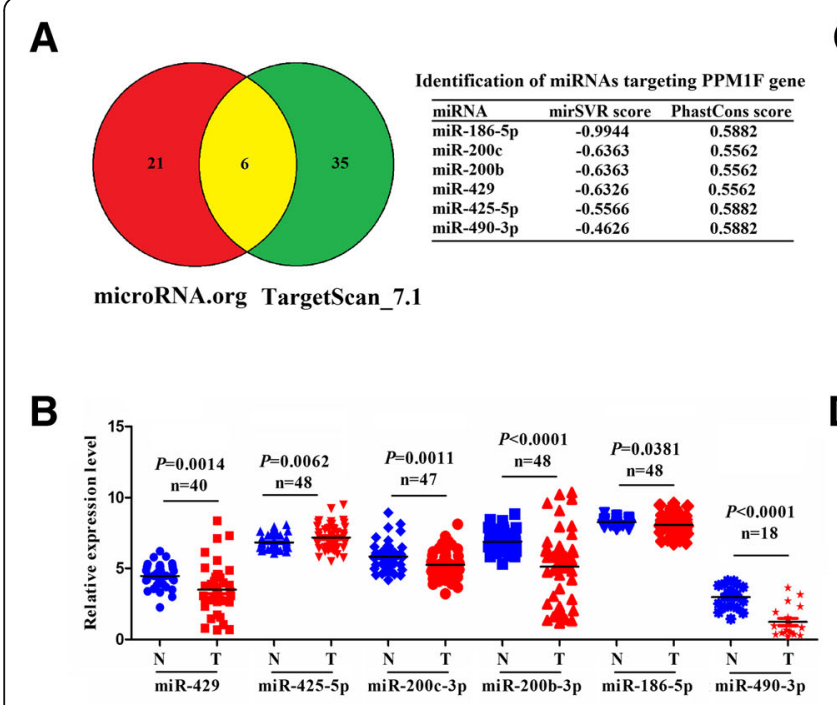

\section{C}

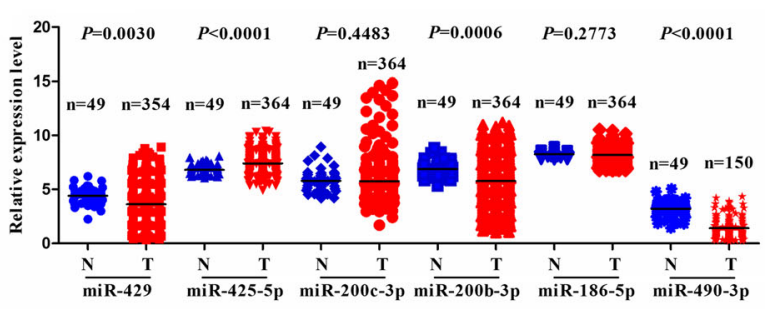

D
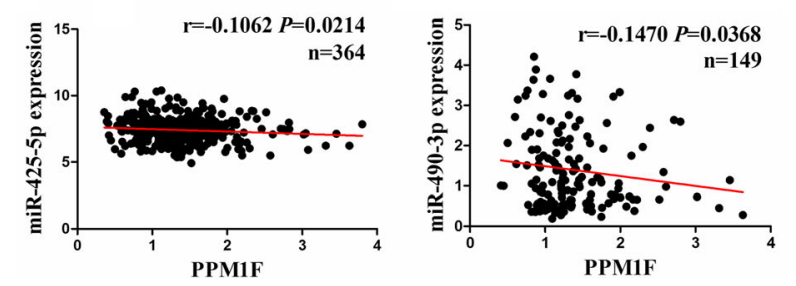

Fig. 2 Identification of the correlation of PPM1F expression with miRNAs in patients with HCC. a Identification of the miRNAs that target PPM1F $3^{\prime}$ UTR by microRNA.org and Targetscan_7.1 prediction tools. b. c TCGA cohort analysis of the expression levels of miRNAs in paired and unpaired HCC tissues. d Pearson's correlation coefficient analysis of the correlation of PPM1F expression with miR-425-5p and miR-490-3p in HCC tissues 
A

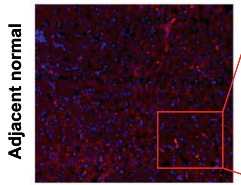

$\underset{\circlearrowleft}{\underline{x}}$

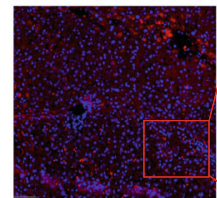

$200 \times$
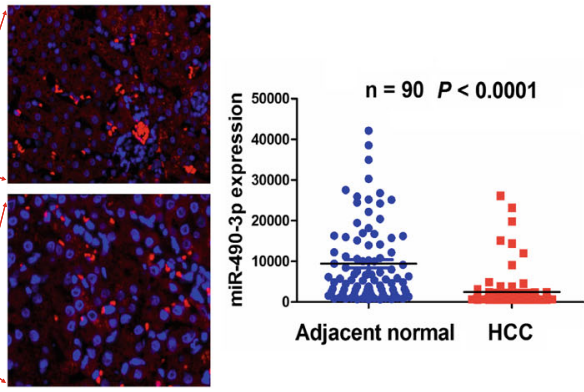

$400 \times$
D

E
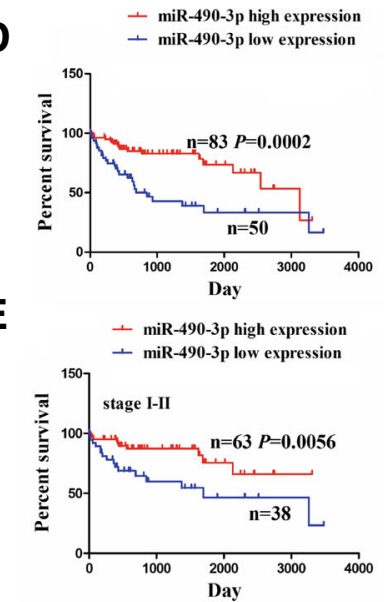

$\mathbf{F}$

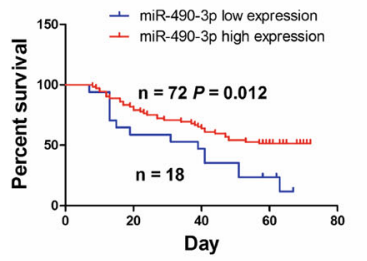

Stage I+ II

C

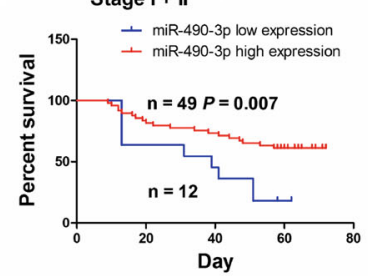

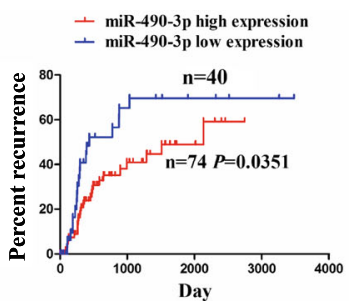

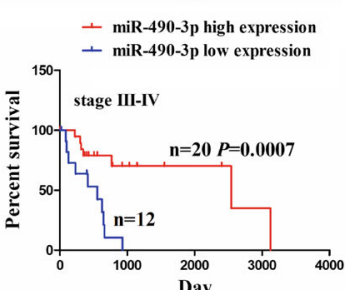

+ miR-490-3p high expression

- miR-490-3p high expression - miR-490-3p low expression

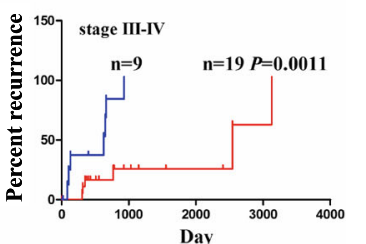

Fig. 3 The association between miR-490-3p expression and the prognosis in patients with HCC. a FISH analysis of the expression level of miR-490-3p in HCC and adjacent normal tissues. b, c Kaplan-Meier analysis of the association of high or low miR-490-3p expression with the overall survival in patients with HCC or those in early stage. $\mathbf{d}$ Kaplan-Meier analysis of the association of high or low miR-490-3p expression with the overall survival and tumor recurrence in patients with HCC in TCGA cohort. e, f Kaplan-Meier analysis of the association of high or low miR-490-3p expression with the overall survival and tumor recurrence in HCC patients in early and late stages

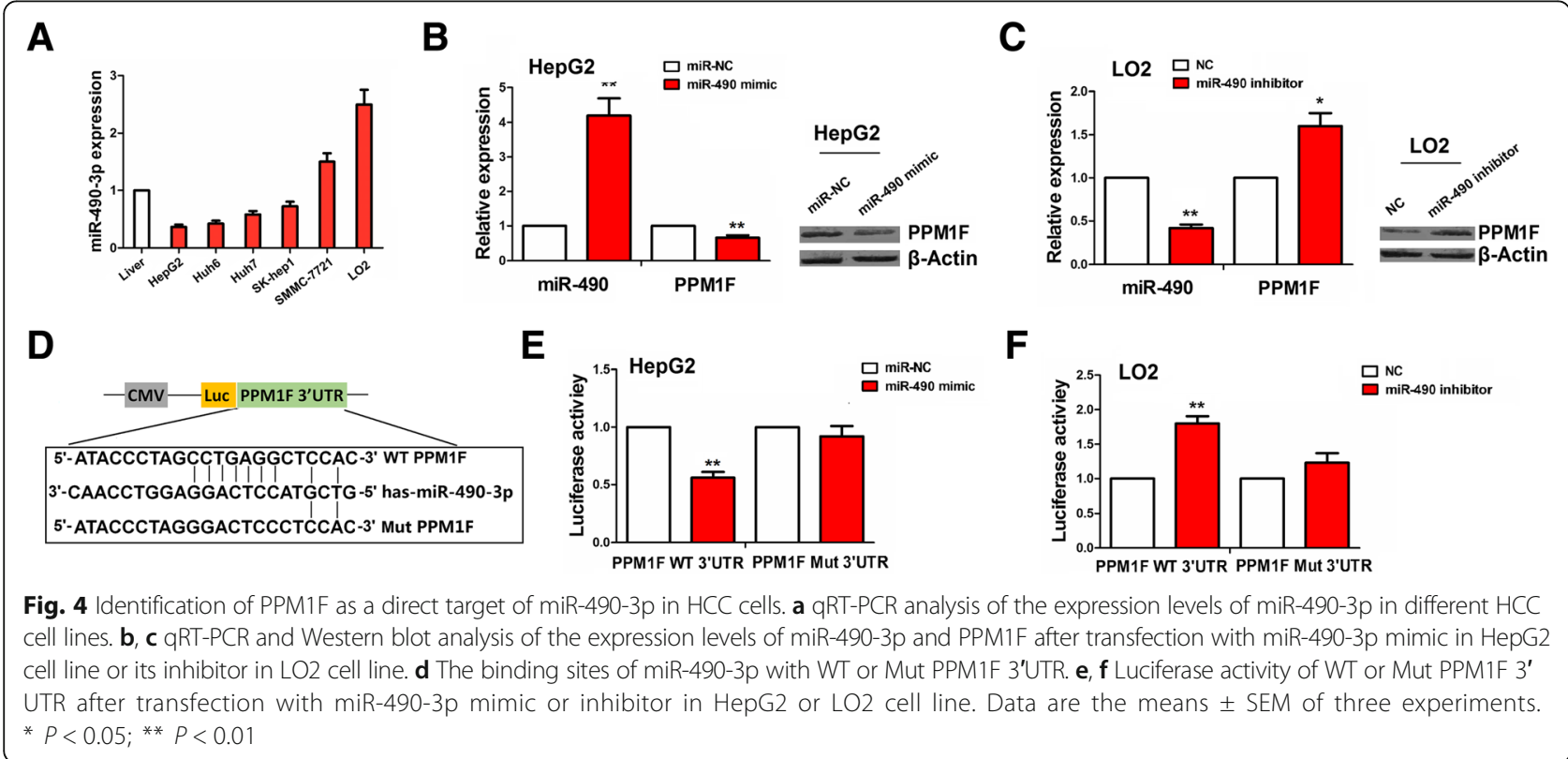


acquired according to its expression levels, survival time and survival status in patients with $\mathrm{HCC}$, and the patients were divided into high PPM1F expression and low PPM1F expression groups (Fig. 1c).

As shown in Additional file 1: Table S2, high expression of PPM1F had no association with the clinicopathological factors (each $P>0.05$ ). Kaplan Meier analysis showed that HCC patients with high PPM1F expression harbored poorer survival $(P=0.0174$, Fig. $1 d)$, but had no difference in tumor recurrence $(P=0.2085$, Fig. 1e) as compared with those with low PPM1F expression. On the basis of TNM stage, the patients in late stage (stage III + IV,$P=0.0372$ ) rather than early stage (stage I + II, $P=0.0847$ ) with high PPM1F expression possessed poorer survival as compared with those with low PPM1F expression (Fig. 1f, g). Furthermore, univariate and multivariate Cox regression analysis revealed that PPM1F expression as well as distant metastasis was an independent prognostic factor of poor survival in patients with HCC (Additional file 1: Table S3).

\section{MiR-490-3p displayed a negative correlation with PPM1F expression in HCC tissues}

To elucidate the reason of PPM1F upregulation in HCC tissues, we investigated the alterations of PPM1F in genetic or epigenetic levels, and found little evidence about the dysregulation of PPM1F at the genetic (Additional file 2: Figure S1A and B) and methylation levels (Additional file 2: Figure S1C), indicating that genetic alterations and methylation modification could not account for the upregulation of PPM1F in HCC.

Whether PPM1F upregulation was caused by miRNAs through the post-transcriptional regulation is needed to be clarified. TargetScan_7.1 and microRNA.org prediction tools were used to identify six miRNAs that may have the potential to bind to PPM1F 3' UTR (Fig. 2a and

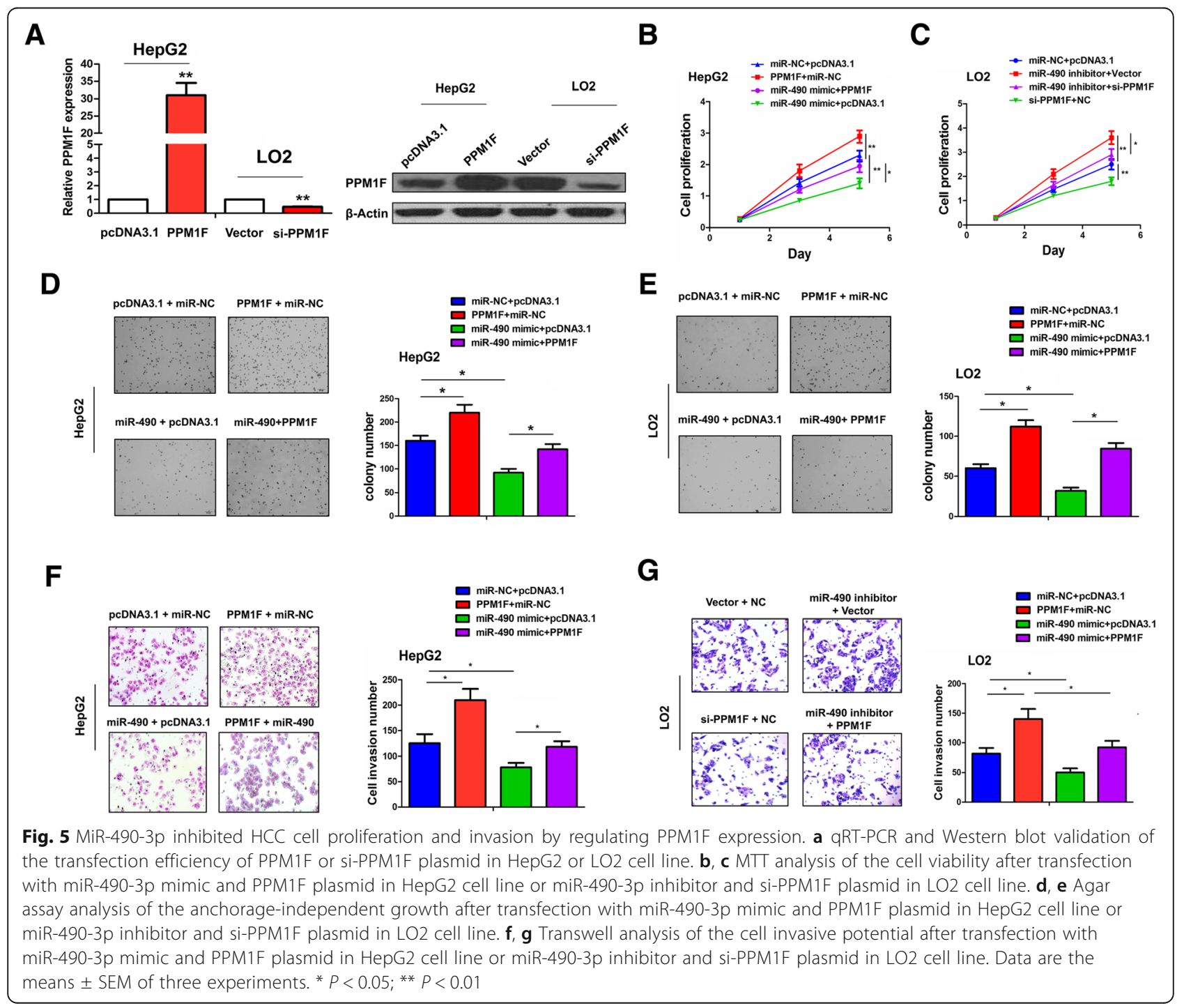


Additional file 1: Table S4, S5). The expression levels of these miRNAs were then examined in paired (Fig. 2b) and unpaired HCC tissues (Fig. 2c), indicating that miR-490-3p had the most significant downregulation in HCC tissues $(P<0.0001)$. Pearson's coefficient analysis showed that miR-490-3p rather than other five miRNAs had the most obviously negative correlation with PPM1F expression $\quad(r=-0.1470, \quad P=0.0368 ; \quad$ Fig. $2 \mathrm{~d}$ and Additional file 3: Figure S2A1-4) and was selected for further observation.

\section{Low expression of miR-490-3p was associated with poor prognosis in patients with $\mathrm{HCC}$}

We further detected the expression levels of miR-490-3p in HCC tissues by FISH and found that miR-490-3p was principally localized in the cytoplasm and dramatically downregulated in HCC tissues as compared with the adjacent non-tumor tissues $(P<0.0001$, Fig. 3a). As shown in Additional file 3: Figure S2B, the cutoff value of miR-490-3p was obtained in our cohort, and we found that, HCC patients with low miR-490-3p expression displayed poorer survival as compared with those with high miR-490-3p expression ( $P=0.012$, Fig. $3 \mathrm{~b})$. The patients in early stage $(P=0.007$, Fig. $3 c)$ rather than in late stage $(P=0.599$, Additional file 3: Figure S2C) with low miR-490-3p expression showed the similar survival prognosis. In TCGA cohort, the cutoff value, sensitivity, specificity and AUC of miR-490-3p were respectively 0.787, 73.3, 59.1\% and 0.63 (Additional file 3: Figure $\mathrm{S} 2 \mathrm{D})$, indicating that miR-490-3p was a promising marker in HCC patients.

As shown in Additional file 1: Table S6, low expression of miR-490-3p had no association with the clinicopathological factors (each $P>0.05$ ) in patients with HCC. Kaplan Meier analysis indicated that HCC patients with low miR-490-3p expression possessed poorer survival $(P$ $=0.0002)$ and higher tumor recurrence $(P=0.0351)$ as compared with those with high miR-490-3p expression (Fig. 3d). The patients in early or late stage with low miR-490-3p expression also had poorer survival $(P=$ $0.0056, P=0.0007$; Fig. $3 \mathrm{e})$ and higher tumor recurrence $(P=0.0033, P=0.0011$; Fig. $3 f)$ as compared with those with high miR-490-3p expression. Moreover, univariate and multivariate Cox regression analysis showed that miR-490-3p expression as well as pathological stage and TNM stage was an independent prognostic factor of poor survival and tumor recurrence in patients with HCC (Additional file 1: Table S7, S8).

\section{PPM1F was identified as a direct target of miR-490-3p in HCC cells}

We examined the expression levels of miR-490-3p in HCC cell lines by qRT-PCR analysis, indicating that miR-490-3p had lower expression in HepG2 cell line

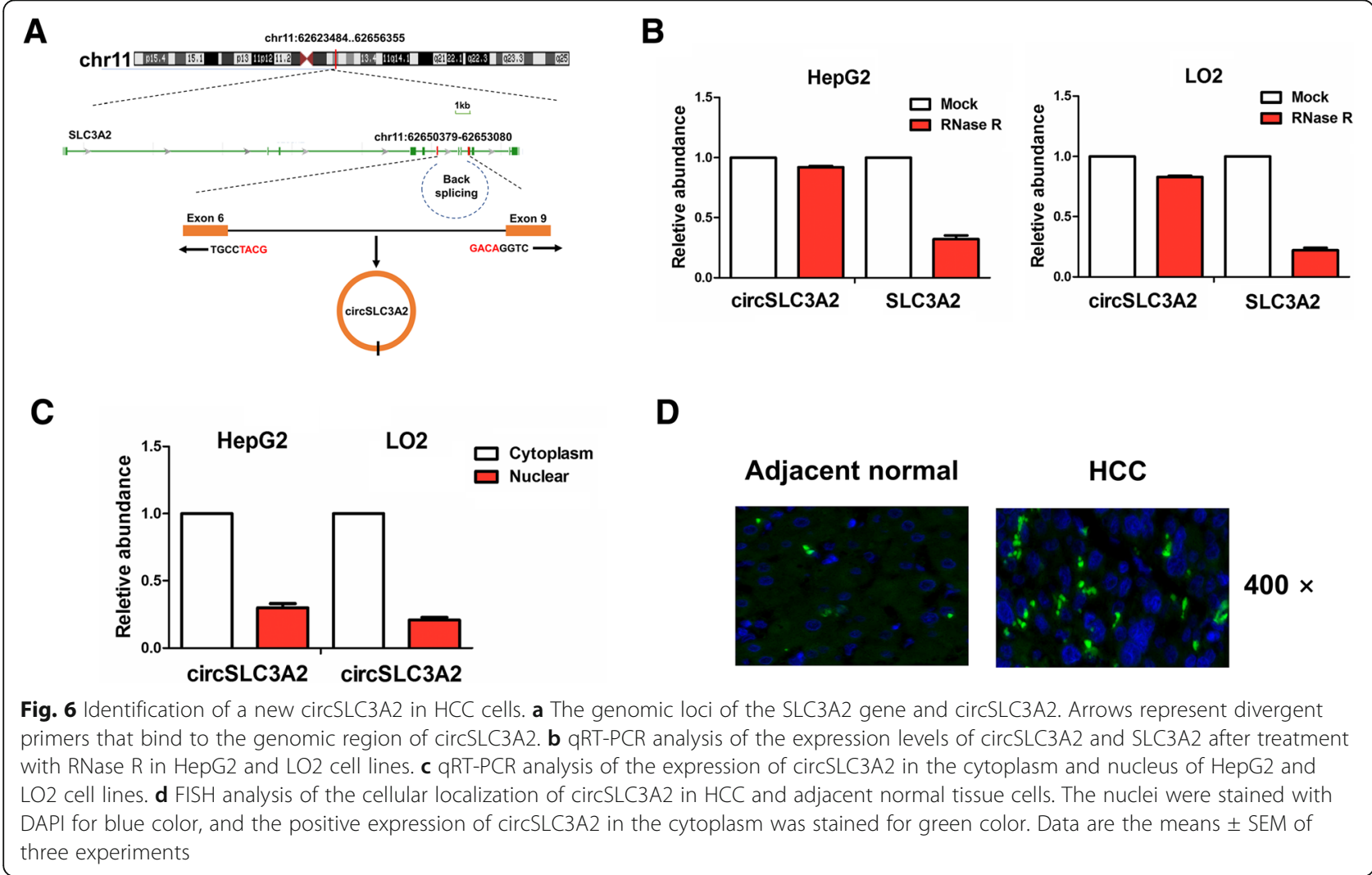


$(P<0.01)$, but higher expression in LO2 cell line $(P<0.05)$ as compared with the normal liver tissue (Fig. 4a). Then, qRT-PCR and western blot analysis showed that miR-490-3p expression was substantially increased, but PPM1F expression was decreased by transfection with miR-490-3p mimic in HepG2 cells $\left({ }^{* *} P<0.01\right.$, Fig. 4b), whereas the opposite results were indicated by transfection with miR-490-3p inhibitor in LO2 cells (" $P<0.05$, "* $P<0.01$; Fig. 4c). Luciferase reporter vectors containing wild type (WT) or mutant (Mut) PPM1F 3'UTR (Fig. 4d) in combination with miR-490-3p mimic or inhibitor were transfected into HepG2 or LO2 cell line. The results showed that the luciferase activity of WT PPM1F 3'UTR was decreased by miR-490-3p mimic in HepG2 cell line $(* * P<0.01$, Fig. 4 e), but increased by miR-490-3p inhibitor in LO2 cell line as compared with the control group ${ }^{* * *} P<0.01$, Fig. 4f). However, the luciferase activity of Mut PPM1F 3'UTR was not affected by miR-490-3p mimic or inhibitor in HepG2 or LO2 cells as compared with the control group $(P>0.05$, Fig. 4e, f).

\section{PPM1F reversed the inhibitory effects of miR-490-3p in HCC cells}

To further define the effects of miR-490-3p on PPM1F expression in HCC cells, we conducted the functional experiments such as MTT, Agar and Transwell assays. The expression levels of PPM1F were validated after transfection with PPM1F or si-PPM1F vector in HepG2 or LO2 cell lines by qRT-PCR and Western blot analysis $\left({ }^{* *} P<0.01\right.$, Fig. 5a). Cell viability was increased by PPM1F overexpression or miR-490-3p inhibitor, but decreased by knockdown of PPM1F or miR-490-3p mimic in HepG2 or LO2 cell line $\left({ }^{*} P<0.05,{ }^{* *} P<0.01\right.$; Fig. 5 b,

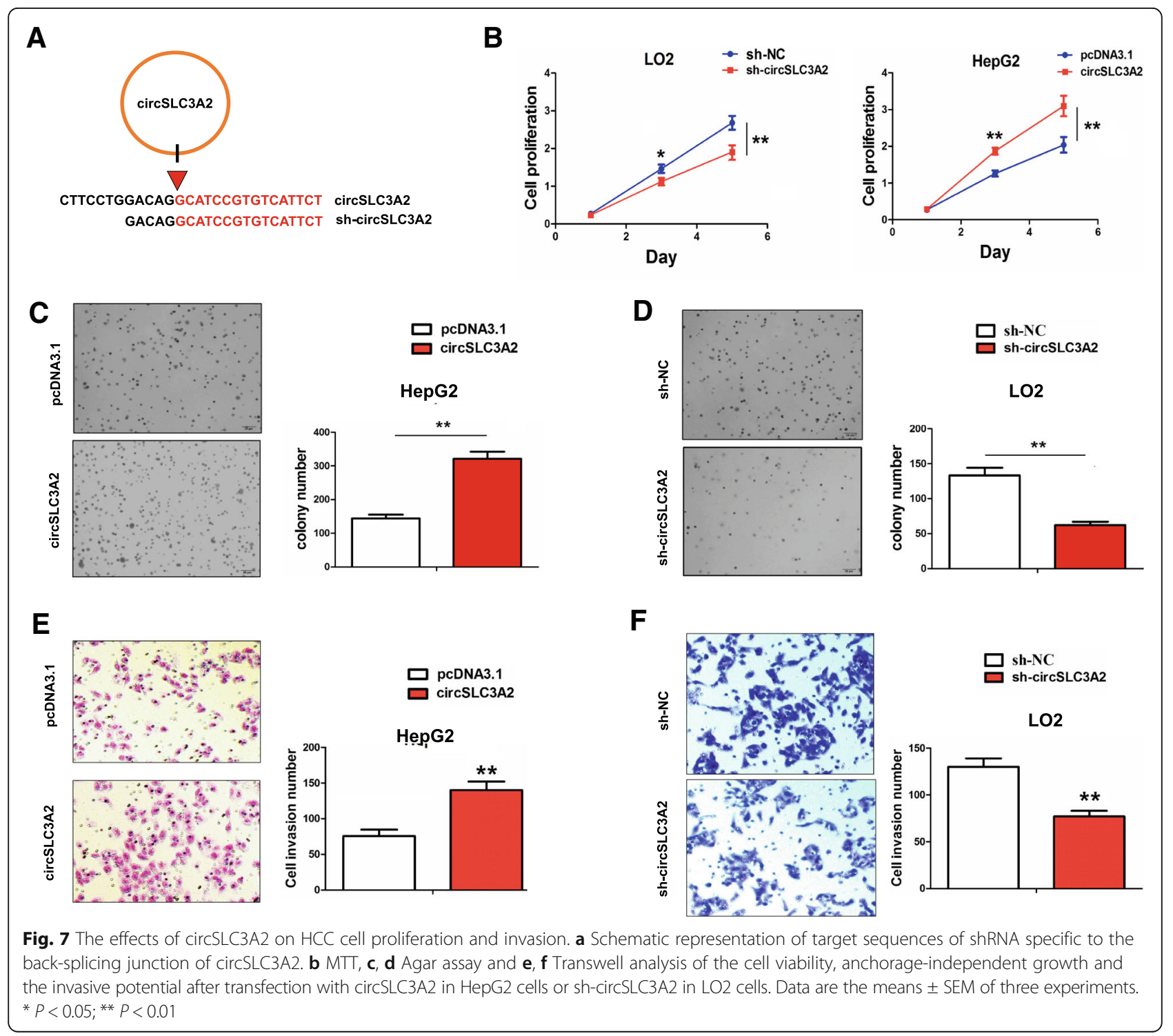


c). Moreover, the anti-proliferative effect induced by miR-490-3p mimic was inversed by ectopic expression of PPM1F in HepG2 cells ( $P<0.05$, Fig. $5 b)$, but the proliferation-promoting effect caused by miR-490-3p inhibitor was counteracted by knockdown of PPM1F in LO2 cells ( ${ }^{* *} P<0.01$, Fig. 5 c). The similar phenomenon for the anchorage-independent growth (" $P<0.05$, Fig. 5 d, e), and cell invasion was shown in Fig. 5 f, g (" $P<0.05)$.

\section{Identification of a new circSLC3A2 in HCC cells}

To identify the circRNAs that can sponge miR-490-3p, we used the circRNAs expression profile and miRNAs prediction software screen out 9 circRNAs that had the potential to bind to the miR-490-3p (Additional file 1: Table S9 and Additional file 4: Figure S3), of which hsa_circ_0022587 might have the greatest potential to sponge miR-490-3p. It was noted that hsa_circ_0022587 is derived from exon 6, 9 regions within solute carrier family 3 member 2 (SLC3A2) locus, and is named as circSLC3A2 (Fig. 6a). According to the qRT-PCR analysis, circSLC3A2 exhibited a resistance to digestion induced by RNase $\mathrm{R}$ exonuclease as compared with the linear SLC3A2 in HepG2 and LO2 cell lines $(P<0.01$, Fig. $6 \mathrm{~b})$. Cytoplasmic and nuclear RNA analysis showed that circSLC3A2 was preferentially localized in the cytoplasm in
HepG2 and LO2 cell lines (Fig. 6c), and the consistent result was shown by FISH in HCC and adjacent normal tissue cells (Fig. 6d).

\section{CircSLC3A2 promoted cell proliferation and invasion in HCC cells}

We devised circSLC3A2 overexpression and shRNA sequences against the back-splicing site of circSLC3A2 (Fig. 7a). The transfection efficiency of circSLC3A2 or sh-circSLC3A2 in HepG2 or LO2 cell line was determined by qRT-PCR analysis $\left({ }^{* *} P<0.01\right.$, Additional file 5 : Figure S4). MTT, Agar and Transwell assays indicated that cell viability $(* * P<0.01$, Fig. $7 \mathrm{~b})$, anchorage-independent growth $(* * P<0.01$, Fig. $7 \mathrm{c}, \mathrm{d})$ and invasive potential $(* * P<$ 0.01 , Fig. 7 e, f) were obviously enhanced by ectopic expression of circSLC3A2 in HepG2 cell line, but attenuated by knockdown of circSLC3A2 in LO2 cell line as compared with the control group.

CircSLC3A2 acted as a sponge of miR-490-3p in HCC cells We established a circSLC3A2 fragment and incorporated it into downstream of the luciferase reporter gene (Fig. 8a). qRT-PCR analysis showed that the expression levels of miR-490-3p were decreased by overexpression of circSLC3A2 in HepG2 cells, but

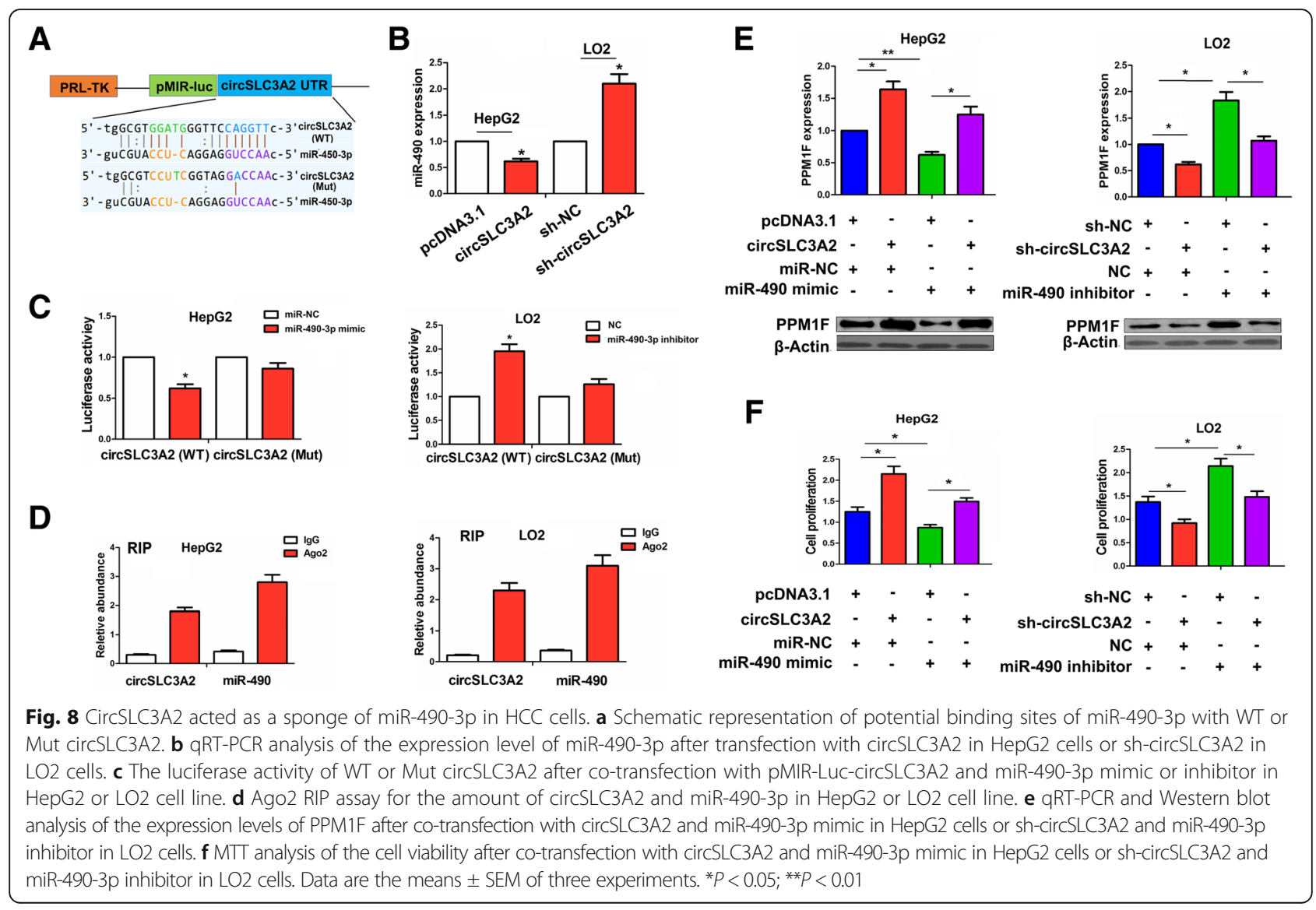


increased by knockdown of circSLC3A2 in LO2 cells (" $P<0.05$, Fig. 8b). The luciferase reporter vector for pMIR-Luc-circSLC3A2 was co-transfected with miR-490-3p mimic or inhibitor into HepG2 or LO2 cell line. The luciferase activity of WT circSLC3A2 was decreased by miR-490-3p mimic in HepG2 cells, but increased by miR-490-3p inhibitor in LO2 cells $\left({ }^{*} P<0.05\right.$, Fig. 8c). However, the luciferase activity of Mut circSLC3A2 was not affected by miR-490-3p mimic or inhibitor in HepG2 or LO2 cell line $(P>$ 0.05). Furthermore, online circular RNA interactome (https://circinteractome.nia.nih.gov/index.html) was used to reveal a high degree of Ago2 occupancy in the region of circSLC3A2 (Additional file 1: Table S10). To validate this result, we conducted RIP assay for Ago2 in HepG2 and LO2 cell lines and examined the expression levels of endogenous circSLC3A2 and miR-490-3p pulled-down from
Ago2-expressed cells by qRT-PCR analysis, indicating that circSLC3A2 and miR-490-3p expression was highly enriched in Ago2 pellet as compared with those in the input control $(P<0.01$, Fig. $8 d)$. We also investigated the expression of PPM1F, a target of miR-490-3p after co-transfection with circSLC3A2 and miR-490-3p mimic into HepG2 cell line or sh-circSLC3A2 and miR-490-3p inhibitor into LO2 cell line by qRT-PCR and Western blot analysis (" $P$ $<0.05,{ }^{* *} P<0.01$; Fig. 8e), indicating that circSLC3A2 overexpression increased the expression of PPM1F, and miR-490-3p mimic reversed this effect; Inversely, knockdown of circSLC3A2 decreased the expression of PPM1F and miR-490-3p inhibitor counteracted this effect. In addition, cell viability, increased by circSLC3A2 overexpression was reversed by miR-490-3p mimic in HepG2 cell line, while cell

A
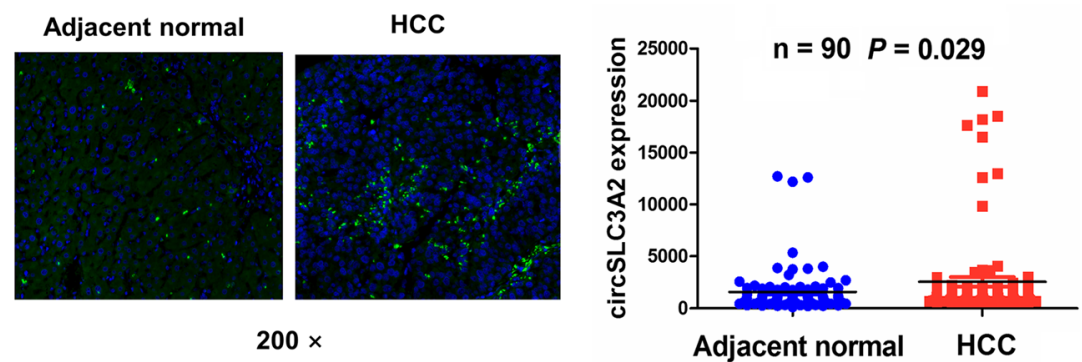

B

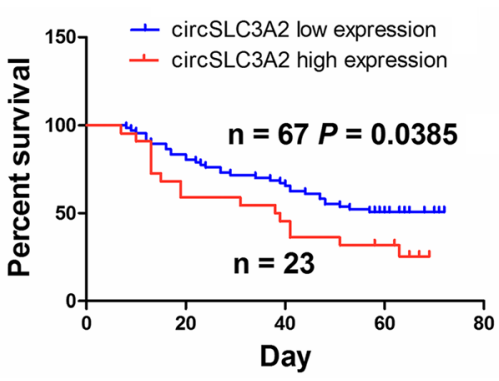

D

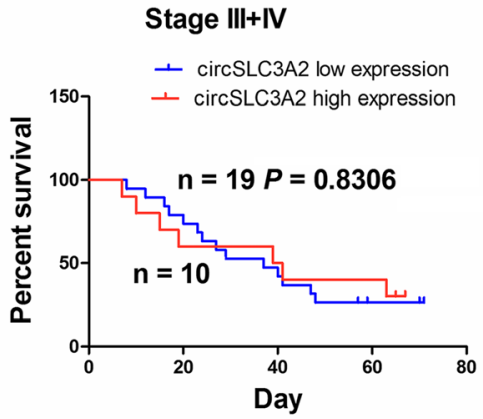

C

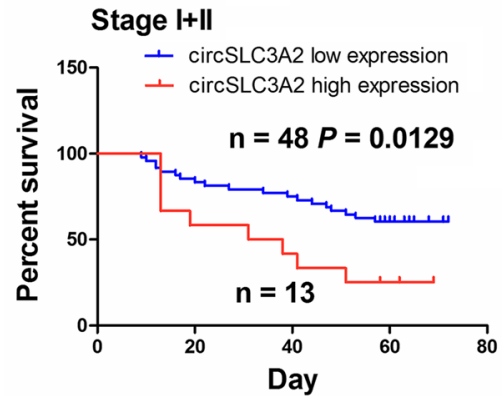

E

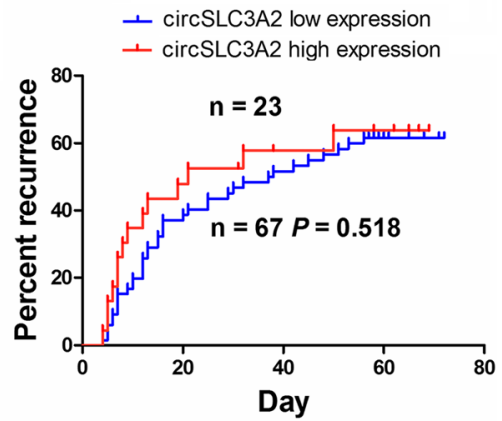

Fig. 9 The association between circSLC3A2 expression and the prognosis in patients with HCC. a FISH analysis of the expression level of circSLC3A2 in HCC and adjacent normal tissues. $\mathbf{b}$ Kaplan-Meier analysis of the association of high or low circSLC3A2 expression with the overall survival in patients with HCC. c, d Kaplan-Meier analysis of the association of high or low circSLC3A2 expression with the survival in HCC patients in early and late stages. e Kaplan-Meier analysis of the association of high or low circSLC3A2 expression with the tumor recurrence in HCC patients 
viability, decreased by knockdown of circSLC3A2 was counteracted by miR-490-3p inhibitor in LO2 cell line $(* P<0.05$, Fig. $8 f)$.

\section{Increased expression of circSLC3A2 was associated with poor survival in patients with HCC}

FISH analysis indicated that the expression of circSLC3A2 was elevated in HCC tissues as compared with the adjacent normal tissues ( $P=0.020$, Fig. 9a), but Pearson correlation analysis showed that circSLC3A2 had no correlation with the miR-490-3p expression in HCC tissues $(r=-0.1477, P=0.0823$, Additional file 6: Figure S5). According to the circSLC3A2 expression levels, overall survival time and status, we obtained the cutoff value of circSLC3A2 by using the cutoff finder in HCC patients, and this value divided the patients into high or low circSLC3A2 expression group. We analyzed the association of circSLC3A2 expression with clinicopathological characteristics and prognosis in patients with $\mathrm{HCC}$, and found that high expression of circSLC3A2 had no association with these parameters (each $P>0.05$ ) (Additional file 1: Table S11). Kaplan-Meier analysis showed that HCC patients as well as those in early stage $(P=0.0385, P=$ 0.0129; Fig. $9 \mathrm{~b}, \mathrm{c})$ rather than in late stage $(P=0.8306$, Fig. 9d) with high circSLC3A2 expression had the poorer survival as compared with those with low circSLC3A2 expression, but the patients with high or low circSLC3A2 expression had no difference in tumor recurrence $(P=$ 0.518, Fig. 9e). Univariate and multivariate Cox regression analyses revealed that circSLC3A2 expression was not an independent prognostic factors of poor survival in patients with HCC (Additional file 1: Tables S12).

\section{Discussion}

Some studies indicate that PPM1F expression is upregulated in cancer tissues and has an association with distant metastasis and poor survival in cancer patients $[5,6]$. But, our previous study showed that, PPM1F expression is downregulated in GC tissues, and loss of PPM1F expression predicts poor survival in patients with GC [8]. In this study, we found that PPM1F expression was increased in HCC tissues and high expression of PPM1F had the positive association with poor survival, acting as an independent prognostic factor in HCC patients or those in late stage. In consistence with the studies [5, 6], our results suggest that PPM1F might be a potential biomarker in HCC patients.

Furthermore, we found that, six miRNAs (miR-186-5p, miR-200c/-b, miR-429, miR-425-5p and miR-490-3p) were identified to have the potential to bind to PPM1F 3'UTR, of which miR-490 expression was dramatically decreased and had the most significant correlation with PPM1F expression in HCC tissues. Our and TCGA cohorts further confirmed that, low miR-490 expression, as an independent prognostic factor, was associated with poor survival and tumor recurrence in HCC patients and those in early or late stage. But, individual study showed that miR-490-3p is upregulated in HCC tissues and cells as compared with the adjacent non-tumor tissues [21]. In coinciding with our results, more studies

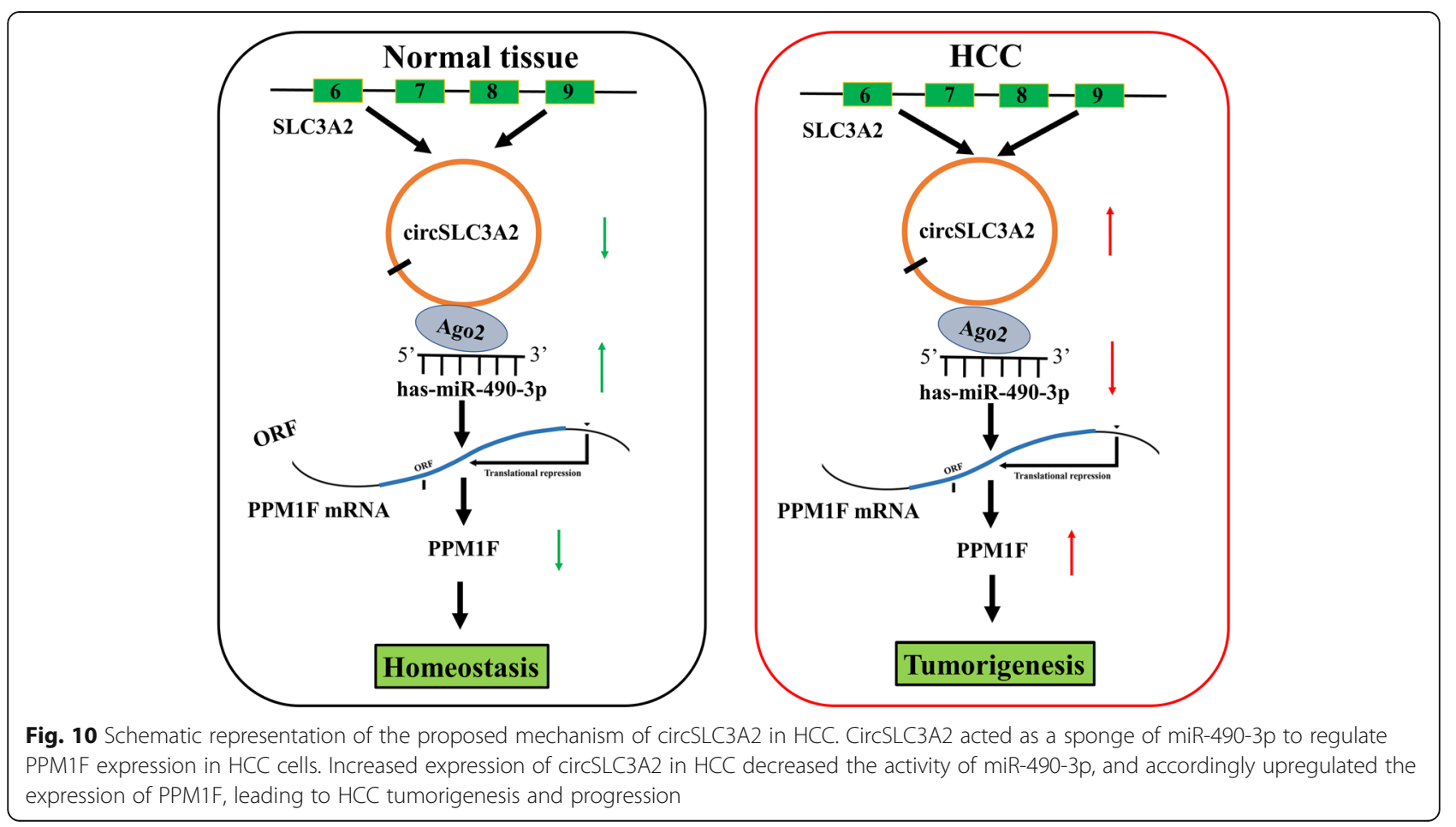


revealed that miR-490-3p is downregulated in colorectal cancer (CRC) [22-24], gastric cancer (GC) [25], HCC [26] and associated with TNM stage and overall survival in CRC patients [23]. These studies indicate that miR-490-3p may be a promising diagnostic biomarker for cancer patients.

Functionally, miR-490-3p has been reported to act as a tumor suppressor in multiple malignancies. It is shown that miR-490-3p inhibits cell growth by inducing apoptosis and cell invasiveness and metastasis by repression of epithelial-to-mesenchymal transition (EMT) through targeting FRAT1, VDAC1 and TGF $\beta R 1$ in CRC cells [22-24], SMARCD1 in GC [25] and BUB1 in HCC [26]. However, miR-490-3p was reported to promote cell growth and invasion via inducing EMT in HCC cells [20]. Our present studies guaranteed the consistent results with a majority of the reports [22-26] and showed the suppressive effects of miR-490-3p on HCC cell proliferation and invasion by targeting PPM1F.

Accumulating data show that circRNAs act as miRNA sponge and induce the dysregulation of miRNAs and their target genes, leading to tumor progression including HCC [20, 27-29]. Hsa_circ_0001649 is regarded as a potential biomarker [30] and circMTO1 and circ_0067934 regulate cell growth and metastasis of HCC by sponging miR-9 and miR-1324 [18, 19]. Moreover, circ_0103809 is shown to promote HCC tumorigenesis by sponging miR-490-5p/SOX2 signaling [31]. We also identified a circRNA derived from SLC3A2 locus, named as circSLC3A2, which appeared to sponge miR-490-3p. SLC3A2 promotes integrindependent renal cancer growth [32] and loss of SLC3A2 inhibits Ras-driven tumorigenesis [33], Furthermore, we found that circSLC3A2, mainly localized in the cytoplasm, was upregulated in HCC tissues, associated with poor survival in patients with $\mathrm{HCC}$, and facilitated cell proliferation and invasion by sponging miR-490-3p and upregulating PPM1F expression, but knockdown of circSLC3A2 reversed these effects (Fig. 10), suggesting that circSLC3A2 might function as an oncogenic factor in $\mathrm{HCC}$ via regulation of the miR-490-3p/PPM1F axis.

\section{Conclusion}

Taken together, our findings demonstrated that low expression of miR-490-3p or high expression of PPM1F was positively associated with poor survival and tumor recurrence in patients with HCC, and miR-490-3p suppressed cell proliferation and invasion by targeting PPM1F. CircSLC3A2 was identified to act as an oncogenic factor in HCC by sponging miR-490-3p and regulating PPM1F expression.

\section{Additional files}

\begin{abstract}
Additional file 1: Table S1. List of the primer sequences. Table S2. The association of PPM1F expression with clinicopathologic characteristics of HCC patients. Table S3. Cox regression analysis of PPM1F expression as survival predictor of HCC patients. Table S4. Identification of miRNAs targeting PPM1F gene by microRNA.org. Table S5. Identification of miRNAs targeting PPM1F gene by TargetScan_7.1. Table S6. The association of miR-490-3p expression with clinicopathologic characteristics of HCC patients. Table S7. Cox regression analysis of miR-490-3p expression as survival predictor of HCC. Table S8. Cox regression analysis of miR-490-3p expression as recurrence predictor of HCC. Table S9. Identification of the circRNAs than sponge the miR-490-3p. Table S10. List of Ago2 occupancy in the region of circSLC3A2. Table S11. The association of circSLC3A2 expression with clinicopathologic characteristics of HCC patients. Table S12. Cox regression analysis of circSLC3A2 expression as survival predictor of HCC. (DOCX $46 \mathrm{~kb}$ )
\end{abstract}

Additional file 2: Figure S1. The association between PPM1F expression and the genetic and methylation alterations in HCC. (A) The genetic alterations of PPM1F in HCC. (B) The alterations of PPM1F in copy number in HCC. (C) The correlation of PPM1F expression with its DNA methylation in HCC. (PDF 1074 kb)

Additional file 3: Figure S2. The association between miR-490-3p expression and the prognosis in patients with HCC. (A1-4) Pearson's correlation coefficient analysis of the correlation of PPM1F expression with miR-429/-200c-3p/-200b-3p/- 186-5p in HCC tissues. (B) ROC curve analysis of the cutoff value, sensitivity, specificity and AUC of miR-490-3p in HCC tissues in our cohort. (C) Kaplan-Meier analysis of the association of high or low miR-490-3p expression with the overall survival of HCC patients in late stage in our cohort. (D) ROC curve analysis of the cutoff value, sensitivity, specificity and AUC of miR-490-3p in HCC tissues in TCGA cohort. (PDF 2903 kb)

Additional file 4: Figure S3. Schematic representation of potential binding sites of miR-490-3p with the 9 circRNAs. (PDF 4573 kb)

Additional file 5: Figure S4. qRT-PCR analysis of the transfection efficiency of circSLC3A2 in HepG2 cells or sh-circSLC3A2 in LO2 cells. Data are the means \pm SEM of three experiments. ${ }^{* *} P<0.01$. (PDF $62 \mathrm{~kb}$ )

Additional file 6: Figure S5. Pearson correlation analysis of the correlation of circSLC3A2 with the miR-490-3p expression in HCC tissues. (PDF $75 \mathrm{~kb}$ )

\section{Abbreviations}

AUC: Area under curve; CircRNA: Circular RNA; CRC: Colorectal cancer; FBS: Fetal bovine serum; FISH: Fluorescence in situ hybridization; GC: Gastric cancer; HCC: Hepatocellular carcinoma; IOD: Immunofluorescence accumulation optical density; MiRNA: MicroRNA; NcRNAs: Non-coding RNAs; PPM1F: Protein phosphatase Mg2+/Mn2+ dependent 1F; qRT-PCR: Quantitative real-time PCR; RIP: RNA immunoprecipitation; ROC: Receiver operating characteristic; TCGA: The Cancer Genome Atlas; TMA: Tissue microarray

\section{Acknowledgements}

This study was supported by Shanghai Science and Technology Commission Western Medicine Guide project (No. 17411966500) and Shanghai Jiao Tong University School of Medicine doctoral innovation fund (No. BXJ201737).

\section{Funding}

This study was supported by Shanghai Science and Technology Commission Western Medicine Guide project (No. 17411966500) and Shanghai Jiao Tong University School of Medicine doctoral innovation fund (No. BXJ201737).

\section{Availability of data and materials}

All data generated or analysed during this study are included in this published article and its additional files.

\section{Authors' contributions}

JZhu and JZhang designed this study and HW drafted the manuscript. HW and WC contributed equally to this work. WC, JZhang, LH, RZ, and XC performed the experiments. MJ and JZhang conducted the statistical analysis. HW wrote 
the paper and JZhang revised the paper. All authors read and approved the final manuscript.

\section{Ethics approval and consent to participate}

The present study was approved by the Hospital's Protection of Human Subjects Committee.

\section{Consent for publication}

Consent for publication has been obtained from the patients.

\section{Competing interests}

The authors declare that they have no competing interests.

\section{Publisher's Note}

Springer Nature remains neutral with regard to jurisdictional claims in published maps and institutional affiliations.

\section{Author details}

${ }^{1}$ The Fifth Department of Digestion, The First Affiliated Hospital of Zhengzhou University, Zhengzhou 450052, People's Republic of China. ${ }^{2}$ Department of Gastroenterology, Shanghai Jiao Tong University Affiliated Sixth People's Hospital, No. 600 Yishan Road, Shanghai 200233, China. ${ }^{3}$ Department of Clinical Medicine, Ningbo University School of Medicine, Ningbo 315211, Zhejiang Province, China. ${ }^{4}$ Department of Gastroenterology, Shanghai Ninth People's Hospital, Shanghai Jiao Tong University School of Medicine, Shanghai, China.

\section{Received: 3 August 2018 Accepted: 1 November 2018}

\section{Published online: 23 November 2018}

\section{References}

1. Siegel RL, Miller KD, Jemal A. Cancer statistics, 2018. CA Cancer J Clin. 2018; 68(1):7-30.

2. Forner A, Reig M, Bruix J. Hepatocellular carcinoma. Lancet. 2018;391(10127): 1301-14.

3. Liu M, Jiang L, Guan XY. The genetic and epigenetic alterations in human hepatocellular carcinoma: a recent update. Protein Cell. 2014:5(9):673-91.

4. Susila A, Chan H, Loh AX, Phang HQ, Wong ET, Tergaonkar V, Koh CG. The POPX2 phosphatase regulates cancer cell motility and invasiveness. Cell Cycle. 2010;9(1):179-87.

5. Tu SH, Lin YC, Huang CC, Yang PS, Chang HW, Chang CH, Wu CH, Chen LC, Ho YS. Protein phosphatase Mg2+/Mn2+ dependent 1F promotes smokinginduced breast cancer by inactivating phosphorylated-p53-induced signals. Oncotarget. 2016:7(47):77516-31.

6. Zhang S, Weng T, Cheruba E, Guo T, Chan H, Sze SK, Koh CG. Phosphatase POPX2 exhibits dual regulatory functions in cancer metastasis. J Proteome Res. 2017;16(2):698-711.

7. Weng T, Koh CG. POPX2 phosphatase regulates apoptosis through the TAK1-IKK-NF-KB pathway. Cell Death Dis. 2017;8(9):e3051.

8. Zhang J, Jin M, Chen X, Zhang R, Huang Y, Liu H, Zhu J. Loss of PPM1F expression predicts tumour recurrence and is negatively regulated by miR590-3p in gastric cancer. Cell Prolif. 2018. https://doi.org/10.1111/cpr.12444 [Epub ahead of print].

9. Klingenberg M, Matsuda A, Diederichs S, Patel T. Non-coding RNA in hepatocellular carcinoma: mechanisms, biomarkers and therapeutic targets. J Hepatol. 2017:67(3):603-18

10. Zhang Q, Song G, Yao L, Liu Y, Liu M, Li S, Tang H. miR-3928v is induced by $\mathrm{HBx}$ via NF-KB/EGR1 and contributes to hepatocellular carcinoma malignancy by down-regulating VDAC3. J Exp Clin Cancer Res. 2018;37(1):14

11. Zhang Z, Zhang $Y$, Sun $X X, M a X$, Chen ZN. microRNA-146a inhibits cancer metastasis by downregulating VEGF through dual pathways in hepatocellular carcinoma. Mol Cancer. 2015;14:5

12. Liu Z, Wang Y, Dou C, Sun L, Li Q, Wang L, Xu Q, Yang W, Liu Q, Tu K. MicroRNA-1468 promotes tumor progression by activating PPAR- $\gamma$-mediated AKT signaling in human hepatocellular carcinoma. J Exp Clin Cancer Res. 2018;37(1):49.

13. Jurmeister S, Baumann M, Balwierz A, Keklikoglou I, Ward A, UhImann S, Zhang JD, Wiemann S, Sahin Ö. MicroRNA-200C represses migration and invasion of breast cancer cells by targeting actin-regulatory proteins FHOD1 and PPM1F. Mol Cell Biol. 2012;32(3):633-51.
14. Luo G, Chao YL, Tang B, Li BS, Xiao YF, Xie R, Wang SM, Wu YY, Dong H, Liu $X \mathrm{D}$, Yang SM. miR-149 represses metastasis of hepatocellular carcinoma by targeting actin-regulatory proteins PPM1F. Oncotarget. 2015;6(35):37808-23.

15. Qu S, Zhong Y, Shang R, Zhang X, Song W, Kjems J, Li H. The emerging landscape of circular RNA in life processes. RNA Biol. 2017;14(8):992-9.

16. Qiu LP, Wu YH, Yu XF, Tang Q, Chen L, Chen KP. The emerging role of circular RNAs in hepatocellular carcinoma. J Cancer. 2018;9(9):1548-59.

17. Yu J, Xu QG, Wang ZG, Yang Y, Zhang L, Ma JZ, Sun SH, Yang F, Zhou WP. Circular RNA cSMARCA5 inhibits growth and metastasis in hepatocellular carcinoma. J Hepatol. 2018;68(6):1214-27.

18. Han D, Li J, Wang H, Su X, Hou J, Gu Y, Qian C, Lin Y, Liu X, Huang M, et al. Circular RNA circMTO1 acts as the sponge of microRNA-9 to suppress hepatocellular carcinoma progression. Hepatology. 2017:66(4):1151-64.

19. Zhu Q, Lu G, Luo Z, Gui F, Wu J, Zhang D, Ni Y. CircRNA circ_0067934 promotes tumor growth and metastasis in hepatocellular carcinoma through regulation of miR-1324/FZD5/Wnt/ $\beta$-catenin axis. Biochem Biophys Res Commun. 2018:497(2):626-32.

20. Zhang J, Liu H, Hou L, Wang G, Zhang R, Huang Y, Chen X, Zhu J. Circular RNA LARP4 inhibits cell proliferation and invasion of gastric cancer by sponging miR-424-5p and regulating LATS1 expression. Mol Cancer. 2017;16(1):151.

21. Zhang LY, Liu M, Li X, Tang H. miR-490-3p modulates cell growth and epithelial to mesenchymal transition of hepatocellular carcinoma cells by targeting endoplasmic reticulum-Golgi intermediate compartment protein 3 (ERGIC3). J Biol Chem. 2013;288(6):4035-47.

22. Zheng $K$, Zhou $X$, Yu J, Li Q Wang $H$, Li M, Shao Z, Zhang F, Luo $Y$, Shen $Z$, Chen $F$, et al. Epigenetic silencing of miR-490-3p promotes development of an aggressive colorectal cancer phenotype through activation of the Wnt/ $\beta$-catenin signaling pathway. Cancer Lett. 2016;376(1):178-87.

23. Liu X, He B, Xu T, Pan Y, Hu X, Chen X, Wang S. MiR-490-3p functions as a tumor suppressor by inhibiting oncogene VDAC1 expression in colorectal cancer. J Cancer. 2018;9(7):1218-30.

24. Xu X, Chen R, Li Z, Huang N, Wu X, Li S, Li Y, Wu S. MicroRNA-490-3p inhibits colorectal cancer metastasis by targeting TGFBR1. BMC Cancer. 2015:15:1023.

25. Shen J, Xiao Z, Wu WK, Wang MH, To KF, Chen Y, Yang W, Li MS, Shin VY, Tong $J H$, et al. Epigenetic silencing of miR-490-3p reactivates the chromatin remodeler SMARCD1 to promote helicobacter pylori-induced gastric carcinogenesis. Cancer Res. 2015;75(4):754-65.

26. Xu B, Xu T, Liu H, Min Q, Wang S, Song Q. MiR-490-5p suppresses cell proliferation and invasion by targeting BUB1 in hepatocellular carcinoma cells. Pharmacology. 2017;100(5-6):269-82.

27. Yang C, Yuan W, Yang X, Li P, Wang J, Han J, Tao J, Li P, Yang H, LV $\mathrm{Q}$, et al. Circular RNA circ-ITCH inhibits bladder cancer progression by sponging miR-17/miR-224 and regulating p21, PTEN expression. Mol Cancer. 2018:17(1):19.

28. Zheng Q, Bao C, Guo W, Li S, Chen J, Chen B, Luo Y, Lyu D, Li Y, Shi G, et al. Circular RNA profiling reveals an abundant circHIPK3 that regulates cell growth by sponging multiple miRNAs. Nat Commun. 2016;7:11215.

29. Huang XY, Huang ZL, Xu YH, Zheng Q, Chen Z, Song W, Zhou J, Tang ZY, Huang $X Y$. Comprehensive circular RNA profiling reveals the regulatory role of the circRNA-100338/miR-141-3p pathway in hepatitis B-related hepatocellular carcinoma. Sci Rep. 2017;7(1):5428.

30. Qin M, Liu G, Huo X, Tao X, Sun X, Ge Z, Yang J, Fan J, Liu L, Qin W. Hsa_ circ 0001649: A circular RNA and potential novel biomarker for hepatocellular carcinoma. Cancer Biomark. 2016;16(1):161-9.

31. Cai H, Hu B, Ji L, Ruan X, Zheng Z. Hsa circ 0103809 promotes cell proliferation and inhibits apoptosis in hepatocell ular carcinoma by targeting miR-490-5p/SOX2 signaling pathway. Am J Transl Res. 2018; 10(6):1690-702.

32. Poettler M, Unseld M, Braemswig K, Haitel A, Zielinski CC, Prager GW. CD98hc (SLC3A2) drives integrin-dependent renal cancer cell behavior. Mol Cancer. 2013;12:169.

33. Estrach S, Lee SA, Boulter E, Pisano S, Errante A, Tissot FS, Cailleteau L, Pons C, Ginsberg MH, Féral CC. CD98hc (SLC3A2) loss protects against ras-driven tumorigenesis by modulating integrin-mediated mechanotransduction. Cancer Res. 2014;74(23):6878-89. 